\title{
Costly Voting when both Information and Preferences Differ: Is Turnout Too High or Too Low?
}

\author{
Sayantan Ghosal and Ben Lockwood* \\ University of Warwick
}

October, 2008

\begin{abstract}
We study a model of costly voting over two alternatives, where agents' preferences are determined by both (i) a private preference in favour of one alternative e.g. candidates' policies, and (ii) heterogeneous information in the form of noisy signals about a commonly valued state of the world e.g. candidate competence. We show that depending on the level of the personal bias (weight on private preference), voting is either according to private preferences or according to signals. When voting takes place according to private preferences, there is a unique equilibrium with inefficiently high turnout. In contrast, when voting takes place according to signals, turnout is locally too low. Multiple Pareto-ranked voting equilibria may exist and in particular, compulsory voting may Pareto dominate voluntary voting. Moreover, an increase in personal bias can cause turnout to rise or fall, and an increase in the accuracy of information may cause a switch to voting on the basis of signals and thus lower turnout, even though it increases welfare.
\end{abstract}

Keywords: Voting, Costs, information, turnout, externality, inefFiCIENCY.

JeL Classification Numbers: D72, D82.

${ }^{*}$ This is a substantially revised version of Department of Economics University of Warwick Working Paper 670, "Information Aggregation, Costly Voting and Common Values", January 2003. We would like to thank B. Dutta, M. Morelli, C. Perrroni, V. Bhaskar and seminar participants at Warwick, Nottingham and the ESRC Workshop in Game Theory for their comments. We would also like to thank the editor and an anonymous referee for their comments. Address for correspondance: Department of Economics, University of Warwick, Coventry CV4 7AL, United Kingdom. E-mails: S.Ghosal@warwick.ac.uk, and B.Lockwood@warwick.ac.uk. 


\section{Introduction}

Many decisions are made by majority voting. In most cases, turnout in the voting process is both voluntary and costly. The question then arises whether the level of turnout is efficient i.e. is there too much or too little voting? In an influential contribution, Borgers (2004) addresses this issue in a model with costly voting and "private values" i.e. where agents preferences in favor of one alternative or the other are stochastically independent. He identifies a negative pivot externality from voting: the decision of one agent to vote lowers the probability that any agent is pivotal, and thus reduces the benefit to voting of all other agents. A striking result of the paper is that the negative externality implies that compulsory voting is never desirable: all agents are strictly better off at the (unique) voluntary voting equilibrium. An implication of this global result is a local one: in the vicinity of an equilibrium, lowering voter turnout is always Pareto-improving.

In this paper, we re-examine the nature of inefficiency of majority voting in a model with costly participation, but where agents differ not only also in innate private values but also in the information that they have about some feature of the two alternatives on offer. Moreover, we assume that agents all have the same preferences over that feature. Such situations arise quite often in the public domain. For example, the two alternatives may be candidates who differ both in competence and policy stance, as in Groseclose (2001). Agents all prefer the more competent candidate, but they disagree on policy stance, and moreover, they all have differing information about the competence of the candidates. Or, the two alternatives may be two different local public goods. As taxpayers, agents all prefer the cheaper public good, but they do not know with certainty which one is cheaper, and moreover, they have personal preferences over the particular public good to be provided.

We know from the Condorcet Jury literature ${ }^{1}$ that when agents differ in the information they have about some feature of the two alternatives that they value in the same way, they will tend to underinvest in information when information is costly ${ }^{2}$. This suggests that with costly voting, Borger's effect may be counteracted - and possibly dominated -

\footnotetext{
${ }^{1}$ Initially, this literature assumed that information was costless and the focus was on how well various voting rules e.g. unanimity or majority aggregate the information in the signals, given that agents behave strategically. Important contributions of this type include Austen-Smith and Banks(1996), Duggan and Martinelli (2001), Feddersen and Pesendorfer(1997), McLennan(1998)).

${ }^{2}$ Information acquisition has been endogenised, by allowing agents to buy signals at a cost prior to voting (Martinelli (2006), Mukhophaya (2003), Persico (2000), Gerardi,D and L.Yariv (2005)). However, without exception, this literature assumes that the act of voting itself is costless and doesn't study the turnout decision.
} 
by a tendency to vote too little, when the commonly valued feature of the two alternatives (competence of the candidate, the cost of the public good) is relatively important.

This paper investigates this issue using the following simple model. Agents must choose between two alternatives. Their preferences between the two depend on an unobserved binary state of the world, plus the randomly determined private preference of the agent in favour of one alternative or the other. The weight, common to all voters, attached to private preferences is $\lambda \in[0,1]$. We interpret $\lambda$ as a measure of the voter's personal bias. When $\lambda=1$, an agent does not care about the state of the world, but only his private preference for one alternative or the other, and when $\lambda=0$, he only cares about the state of the world. Prior to the decision to vote, agents simultaneously observe private informative signals about the state of the world ${ }^{3}$

Voting is costly, and agents differ in their voting costs, e.g. the cost of attending meetings or going to the polling station. Voting costs are privately observed. All agents simultaneously decide whether they want to abstain or incur the voting cost and vote for one of the two alternatives, which is chosen by majority vote ${ }^{4}$. So, when agents do not care about the state of the world, our model reduces to Borgers'(2004) model, and when agents only care about the state of the world, our model is close to a symmetric version of Martinelli (2006).

The focus of our paper is both on whether agents vote (the equilibrium turnout probability) and how they vote i.e. whether they use their information or not when voting. We begin by characterizing the voting decision, conditional on turnout. We show that independently of the numbers of agents who have decided to participate, within the class of symmetric strategies, there is a (generically) unique weakly undominated Bayes-Nash equilibrium of the voting sub-game. If $\lambda<\hat{\lambda}$, all voters will vote according to their signal i.e. vote for the alternative that is best in the state of the world that is forecast to be more likely, given their signal. If $\lambda>\hat{\lambda}$, all voters will vote according to the alternative that they are biased in favour of (in what follows, their private value). Moreover, $\hat{\lambda}$ is increasing in the accuracy of the signal and in the limit $\hat{\lambda}$ tends to a half. If $\lambda<\hat{\lambda}$, there is some (inefficiently low) information aggregation: if $\lambda>\hat{\lambda}$, this information is not used at all.

We then study the turnout decision. We focus on symmetric equilibria where agents turnout to vote iff their cost of doing so is below some critical value. If subsequent voting

\footnotetext{
${ }^{3}$ For the most part, we assume that signals are costless (the case of costly signals is dealt with briefly in Section 6.1 below).

${ }^{4}$ In the event of a tie, each alternative has an equal probability of being chosen.
} 
is according to private values, i.e. $\lambda>\hat{\lambda}$, all agents will behave in the same way i.e. there will be some equilibrium critical turnout cost $c^{*}$ below (above) which every voter will (not) vote. But, if subsequent voting is according to signals, i.e. $\lambda<\hat{\lambda}$, the turnout decision is more subtle: the equilibrium critical turnout cost at which an agent is indifferent about turnout will depend on whether the private value and the signal match or not. This cutoff is higher if there is agreement $\left(c_{M}^{*}>c_{D}^{*}\right.$, in obvious notation, where $M$ denotes the situation where the private value and the signal match and $D$ the situation where the two do not match).

To interpret this result, think about the local public good example. When the payoff from the local public good relative to money is relatively high, there is a unique symmetric equilibrium where those who turnout, vote for their most preferred public good. When this relative payoff is low, in any equilibrium, those who turnout, vote for the public good that they believe to be cheapest. An agent whose privately preferred public good is also cheapest has a higher turnout probability than an agent whose privately preferred public good is the costlier one.

We also show that there is a discontinuity in the turnout probability: as $\lambda$ drops below the critical value, causing agents to switch from voting according to private values to voting according to signals, the turnout probability drops discontinuously. This is because when voting is according to signals, agents have an incentive to free-ride on the turnout of others, as is well-known. This has an important implication. As already remarked, when the accuracy of the signal increases, this increases $\hat{\lambda}$, and this can cause voting behavior to switch to signals, thus lowering the turnout probability. Thus, we find that better information can lower turnout. This is similar to a recent finding by Taylor and Yildirim (2005), but the mechanism at work is rather different.

A key issue is the efficiency of the turnout decision. In addition to the negative "pivot" externality identified by Borgers (2004), in our model, there is a positive informational externality: an individual voter, by basing his voting decision on his informative signal, improves the quality of the collective decision for all voters. Our main finding - and thus the main result of the paper - is that which externality dominates depends entirely on which voting equilibrium prevails. Specifically, if $\lambda>\hat{\lambda}$, so that voting is according to private values, turnout is inefficiently high: a small coordinated decrease in $c^{*}$ makes all agents better off ex ante. If $\lambda<\hat{\lambda}$, so that voting is according to signals, turnout is inefficiently low, whether a voters' signal agrees with his private value or not: that is, a small coordinated increase in $c_{M}^{*}$ or $c_{D}^{*}$ makes all agents better off ex ante. Note that these welfare results are more subtle than they may first appear. First, the fact that $c_{M}^{*}$ is too low is quite surprising, as in some sense, agents whose signal and private value match 
are "overmotivated" to vote. Second, there can be too much voting even if the weight attached to private values is quite high $(\lambda \simeq 0.5)$.

Some additional welfare results follow in the case where voting is according to the signal, at least for the special case where there is zero weight on the private value $(\lambda=0)$. For a finite and small electorate, (a) voting equilibria with a higher turnout may Paretodominate other voting equilibria with a lower turnout, and (b) compulsory voting may Pareto-dominate voluntary majority voting.

In the next section, we set out the model. Section 3 characterizes turnout equilibria. Section 4 contains the main results on efficiency of equilibrium. Sections 5 discusses the comparative statics of increased signal informativeness and personal bias. The last section discusses possible extensions, related literature, and concludes. Proofs are gathered together in the appendix.

\section{The Model}

There is a set $N=\{1, . . n\}$ of agents, who can collectively choose between two alternatives, $A$ and $B$. Agents have payoffs over alternatives that depend on both the state of the world and their own private preferences for one alternative or another. In particular, we assume ${ }^{5}$ that the payoff from alternative $L \in\{A, B\}$ is

$$
(1-\lambda) v(L, s)+\lambda u\left(L, t^{i}\right), \lambda \in[0,1]
$$

where $s \in\left\{s_{A}, s_{B}\right\}$ is the state of the world, and $t^{i} \in\{A, B\}$ is a variable determining $i^{\prime} s$ favoured alternative (private value). Then $u\left(L, t^{i}\right)=1$ if $t^{i}=L$, and 0 otherwise, and $v\left(A, s_{A}\right)=v\left(B, s_{B}\right)=1$ and 0 otherwise.

Some possible interpretations of this set-up are as follows. First, the alternatives could be candidates, who will, if elected, implement predictable policies (as in the citizencandidate model). In addition, only one of the candidates is "good" e.g. honest or competent, i.e. $K$ is good if $s=s_{K}$. The higher $\lambda$, then, the more voters weight policy over competence. This kind of model has been widely studied e.g. Groseclose (2001).

Second, $A$ and $B$ could be two types of discrete local public goods e.g. libraries, swimming pools, funded by a uniform head tax. One of these is more costly than the other, and moreover, voters have some individual preference for one or the other measured by their type $t^{i}$. If the head tax needed for the cheaper good is normalized to zero, and

\footnotetext{
${ }^{5}$ For tractability, we focus on the special case where the payoff is additive in $(L, s)$ and $\left(L, t^{i}\right)$. The case where $w$ is multiplicative is studied in an earlier version of this paper, available on request.
} 
that for the expensive one to 1, in units of a private good, and utility is quasi-linear in that private good, then payoffs over the two projects are, up to a linear transformation ${ }^{6}$, given by (2.1). Then, $\lambda$ measures the marginal willingness to pay for the public good relative to the private good.

The sequence of events is as follows.

Step 0. The state of the world is realized, and each $i \in N$ privately observes his type $t^{i}$, his voting $\operatorname{cost} c^{i}$, and and informative signal about the state of the world, $\sigma^{i} \in\{A, B\}$.

Step 1. Each $i \in N$ decides whether to turn out (attend a meeting, go to the polling station) at a cost of $c^{i}$.

Step 2. All those who have decided to turn out, vote for either $A$ or $B$.

Step 3. The alternative with the most votes is selected. If both $A, B$ get equal numbers of votes, each is selected with probability 0.5 .

The distributional assumptions are as follows. Variables $\left(t^{1}, t^{2}, \ldots, t^{n}\right)$ are i.i.d. random draws from a distribution on $\{A, B\}$ with $\operatorname{Pr}\left(t^{i}=A\right)=0.5$. Agents all believe that each $s$ in $\left\{s_{A}, s_{B}\right\}$ is equally likely. Signals are informative: the probability of signal $\sigma^{i}=L$, conditional on state $s_{L}$ is $q>0.5, L=A, B$. Conditional ${ }^{7}$ on $s$, the $\left(\sigma^{1}, . . \sigma^{n}\right)$ are i.i.d. The $\left(c^{1}, c^{2}, . . c^{n}\right)$ are i.i.d.: $c^{i}$ is distributed on support $[\underline{c}, \bar{c}] \subset \Re_{+}$with the probability distribution $F(c)$. Finally, we also assume that for any $i, c^{i}, t^{i}, s$ (and therefore, $\sigma^{i}$ ) are mutually independent.

Our equilibrium concept is Bayesian equilibrium, with three additional relatively weak assumptions $^{8}$. First, we suppose all agents behave alike in equilibrium (symmetry). Second, we rule out randomization ${ }^{9}$. Third, we assume that player's equilibrium strategy is admissible (weakly undominated).

Some comments are now in order. First, the assumption that voters only observe noisy signals about the state, while motivated by the Condorcet Jury literature, sits quite comfortably with the two interpretations of our model offered above. It is quite plausible that members of the electorate know something, but not everything about candidate

\footnotetext{
${ }^{6}$ For example if $L$ is the cheap project, $i^{\prime} s$ payoff is some $\beta u\left(L, t^{i}\right)$, and if it is the expensive project, $i^{\prime} s$ payoff is $-1+\beta u\left(L, t^{i}\right)$. Adding 1 to both payoffs, dividing through by $1+\beta$, and setting $\lambda=\frac{\beta}{1+\beta}$, and interpreting $s_{L}$ as the event that project $L$ is cheap, gives (2.1).

${ }^{7}$ Of course, the signals are unconditionally correlated (indeed, affiliated).

${ }^{8}$ We are following Borgers(2004) in making these three assumptions.

${ }^{9}$ In Condorcet Jury models, this is generally a too strong assumption, as a Bayes-Nash equilibrium in pure strategies generally does not exist (Austen-Smith and Banks(1996), Wit(1998)). Howoever, due to the symmetry of our model and the voting rule of majority voting, a pure-strategy equilibrium always exists.
} 
competence, or about the costs of public good provision, and furthermore, it is equally plausible that their information is heterogenous.

Second, at Step 1, we assume that an agent who have chosen to turn out does not observe the total number of voters. This describes a situation where the electorate is large and does not physically meet during the electoral process. If instead, voting takes place at a meeting of some kind, then it is reasonable to suppose that voter $i$ observes the number of other voters who have chosen to participate excluding themselves, which we denote by $l$. In this case, $i^{\prime} s$ information set is $\left(t^{i}, c^{i}, \sigma^{i}, l\right)$. As we argue in section 3.1, it makes no difference which of these assumptions hold: there is always a unique equilibrium at the voting stage.

Third, note that the information $\sigma^{i}$ about the state of the world arrives before the turnout decision has been made. This seems realistic. Prior to voting in a general election, for example, voters would get some information from the mass media, personal contact with the candidates, etc. In practice, this information generally arrives prior to the decision to go to the polling station.

Finally, note that when $\lambda=1$, our model reduces to that of Borgers (2004). In that case, the information from the signal is irrelevant, as agents will obviously always vote for the alterative indicated by $t^{i}$ i.e. vote according to their private value, whatever $\sigma^{i}$. Also, when $\lambda=0$, our model is very close to a symmetric version of Martinelli's(2006) model of costly information acquisition ${ }^{10}$, because in this case, costly voting and costly acquisition are identical obstacles to voting (an agent who cares only about the state of the world will never acquire information about the state unless he intends to use it).

\section{Equilibrium}

We begin by characterizing best responses at stage 2 , the voting stage. We, then, characterize the participation decision.

\subsection{Voting}

At this stage, a strategy for $i$ maps his preference type and information $\left(t^{i}, \sigma^{i}\right)$, into a decision to vote for $A$ or $B$. Say that a voter votes according to her private value if he votes for alternative $t^{i}$, whatever $\sigma^{i}$. Say that a voter votes according to her signal if he

\footnotetext{
${ }^{10}$ His model differs in some details (information is continuous, not binary as here, the cost of information is non-stochastic, unlike here) and in the focus, which is mostly on asympotitc results as the number of agents gets large.
} 
he votes for alternative $\sigma^{i}$, whatever $t^{i}$. Then, we have a key intermediate result:

Lemma 1. There is a critical value $0<\hat{\lambda}<1$, where $\hat{\lambda}=(q-0.5) / q$, such that (i) if $\lambda \geq \hat{\lambda}$, there is always a symmetric best response at the voting stage where those who turnout vote to according to their private value; (ii) if $\lambda \leq \hat{\lambda}$, there is always a symmetric best response at the voting stage where those who turnout vote according to their signal. Moreover, these are the only symmetric best-responses in admissible, pure strategies when $\lambda \neq \hat{\lambda}$.

This result is intuitive; agents vote on the basis of their private value only if their private value is a sufficiently important component of preferences. Information aggregation occurs if and only if $\lambda \leq \hat{\lambda}$. Otherwise, the outcome is the same as if the voters observed no signals. So, in the local public good example, agents vote for the project that they believe to be cheaper iff $\lambda \leq \hat{\lambda}$; if $\lambda \geq \hat{\lambda}$, they vote for the one that they prefer the most.

Note the decision to vote according to private values or signals is inefficient for two reasons, because a voter makes the calculation conditional on the event that he is pivotal. First, he overestimates the benefit from voting according to private values; ex ante, he will be pivotal with probability less than 1 . Second, he ignores the external benefit for other voters of voting according to his signal. Thus, it can be shown that there is a critical value $\tilde{\lambda}>\hat{\lambda}$, such that if $\tilde{\lambda}>\lambda>\hat{\lambda}$, all voters can be made better off by switching to voting according to signals ${ }^{11}$.

Note also that when voters vote according to their signals, they do so non-strategically i.e. ignoring any information inferred from the fact that they are pivotal. This is because strategic voting in Condorcet Jury type models - of which this is one - arises only when the two alternatives are asymmetric in some way, and here everything is symmetric (technically, all the hypotheses of Theorem 1 of Austen-Smith and Banks (1996) are satisfied in our model).

Finally, note that Lemma 1 generalizes directly to the case where each voter observes the number of other voters. It is easy to see that this different information structure makes no difference to the equilibrium outcome. This is because by the proof of Lemma $1, \hat{\lambda}$ depends only on $q$ (and not $l$, the number of other participants).

\footnotetext{
${ }^{11}$ Specifically, the ex ante benefit of voting according to signals is $(1-\lambda) Q+0.5 \lambda$, and the ex ante benefit of of voting according to private values is $(1-\lambda)[\phi+(1-\phi) 0.5]+0.5 \lambda$, where $Q$ is the probability that the alternative chosen matches the state of the world, and $\phi$ is the probability that any voter is pivotal, both of which depend on $l$. These two are equal at $\tilde{\lambda}=\frac{Q-0.5}{Q+0.5 \phi-05}$. As $Q>q, \phi<1, \tilde{\lambda}>\hat{\lambda}$.
} 


\subsection{The Benefits of Voting}

We now turn to stage 2, the turnout decision. Turnout is determined by weighing the benefits of voting against the costs. In this section, we characterize the benefits.

Lemma 2. If $\lambda>\hat{\lambda}$, i.e. voting is according to private values, the expected benefit ${ }^{12}$ to voting, conditional on $t^{i}$, and given that exactly $l$ other agents turnout is

$$
b_{P}(l)=\left\{\begin{array}{cc}
\lambda 0.5 g\left(\frac{l}{2}: l, 0.5\right), & l \text { even } \\
\lambda 0.5 g\left(\frac{l+1}{2}: l, 0.5\right), & l \text { odd }
\end{array}\right.
$$

where $g(m: n, \pi)$ is the Binomial probability ${ }^{13}$ of $m$ successes in $n$ trials with success probability $\pi$.

This can be explained intuitively as follows. Any agent who has decided to vote is pivotal only in two situations. The first is where the number of other voters, $l$, is even, and half of the voters vote for each alternative. If voters are voting according to private values, from voter $i^{\prime} s$ perspective, other voters are equally likely to vote for either alternative, so voter $i$ assesses his pivot probability at $g\left(\frac{l}{2}: l, 0.5\right)$. The second is where the number of voters is odd, and where the numbers of other votes for the two alternatives only differ by one. In this case, voter $i$ assesses his pivot probability at $g\left(\frac{l+1}{2}: l, 0.5\right)$. So, (3.1) says that the benefit of voting, conditional on $l$, is just proportional to the pivot probability.

Now when $\lambda>\hat{\lambda}$, let $B_{P}(p)$ be the expected benefit to voting, given that all other agents turnout with probability $p$. This is simply the expected value of $b_{P}(l)$, given that $l$ agents turnout with probability $g(l: n-1, p)$;

$$
B_{P}(p)=\sum_{l=0}^{n-1} b_{P}(l) g(l: n-1, p)
$$

Now suppose that $\lambda<\hat{\lambda}$, so that all agents vote according to signals. In this case, we calculate the benefit to voting in a slightly different way. Denote by $Q(l)$ the probability that the alternative chosen matches the state if exactly $l$ agents vote according to their signals. This is simply the probability that at least half the agents get a signal that matches the state, remembering to multiply by 0.5 if exactly $\frac{l}{2}$ get the right signal:

$$
Q(l)=\left\{\begin{array}{cc}
\sum_{k=(l+1) / 2}^{l} g(k: l, q) & \text { if } l \text { is odd } \\
\sum_{k=\frac{l}{2}+1}^{l} g(k: l, q)+0.5 g\left(\frac{l}{2}: l, q\right) & \text { if } l \text { is even }
\end{array}\right.
$$

\footnotetext{
${ }^{12}$ Both in the statement of Lemma 1 and in what follows, the phrase "benefit of voting" refers to the expected payoff from voting net of the payoff from not voting.

${ }^{13}$ That is $g(m: n, \pi)=\left(\begin{array}{c}n \\ m\end{array}\right) \pi^{m}(1-\pi)^{n-m}$.
} 
Say agent $i$ is a $M$-agent if $\sigma^{i}=t^{i}$ i.e. the signal and the private value match, and a $D$-agent if $\sigma^{i} \neq t^{i}$ i.e. the signal and the private value do not match. Let $b_{M}(l)$ (resp. $\left.b_{D}(l)\right)$ denote the expected benefit to voting for a M-agent (resp. D-agent), given that exactly $l$ other agents turn out, when voting is according to signals.

Lemma 3. If $\lambda<\hat{\lambda}$, i.e. voting is according to signals, the expected benefits to voting, given that exactly $l$ other agents turn out are

$$
b_{M}(l)=Q(l+1)-Q(l), b_{D}(l)=(1-2 \lambda)(Q(l+1)-Q(l))
$$

The interpretation is that $Q(l+1)-Q(l)$ is the increase in the probability that the alternative chosen matches the state if some agent $i$ votes, given that exactly $l$ other agents also vote. The benefit is scaled down for a D-agent by the factor $0<1-2 \lambda<1$, because when the alternative chosen matches the state, it must clash with his private preference $(0<1-2 \lambda$ because $\lambda \leq \hat{\lambda}<0.5)$.

Now let $B_{M}(p), B_{D}(p)$ be the expected benefits to voting for M-agent and D-agent respectively, given that all other agents turnout with probability $p$. These are simply the expected values of $b_{M}(l), b_{D}(l)$, given that $l$ agents turnout with probability $g(l: n-1, p)$;

$$
B_{k}(p)=\sum_{l=0}^{n-1} b_{k}(l) g(l: n-1, p), k=M, D
$$

Combining (3.4) and (3.5), we see that

$$
B_{M}(p)=Q_{+}(p)-Q(p), B_{D}(p)=(1-2 \lambda)\left(Q_{+}(p)-Q(p)\right)
$$

where

$$
Q(p)=\sum_{l=0}^{n-1} f(l: p, n-1) Q(l), Q_{+}(p)=\sum_{l=0}^{n-1} f(l: p, n-1) Q(l+1)
$$

So, $Q(p)$ is the probability that the alternative chosen matches the state, given that some $i$ does not vote and the remaining $n-1$ agents vote with probability $p ; Q_{+}(p)$ is the same probability, but assuming instead that $i$ does vote. Note that as $\lambda<\hat{\lambda}<$ $0.5, B_{M}(p), B_{D}(p)$ are always both positive.

To develop some intuition, and for later use in examples, we calculate $Q_{+}(p)-Q(p)$ explicitly when $n=2$ and when $n=3$. It is shown in the Appendix that when $n=2$,

$$
Q_{+}(p)-Q(p)=(q-0.5)(1-p)
$$

so that, intuitively, the benefit to voting according to signals is increasing in the accuracy of the signal, $q$, and decreasing in $p$, the probability that the other agent votes. When 
$n=3$,

$$
Q_{+}(p)-Q(p)=(q-0.5)\left[2 p^{2} q(1-q)+(1-p)^{2}\right]
$$

In this case, $Q_{+}(p)-Q(p)$ is no longer monotone in $q$ or $p$; as we shall see, this nonmonotonicity can generate multiple equilibria.

\subsection{Equilibrium in Cutoff Strategies}

We are now in a position to define an equilibrium in cutoff strategies. First consider the case $\lambda>\hat{\lambda}$. If all other agents turnout with probability $p$, then $i^{\prime} s$ (strict) best response is to turn out iff $c^{i} \leq B_{P}(p)=\tilde{c}$ where $\tilde{c}$ is a cutoff. Following Borgers, we call this a cutoff strategy. Then, an equilibrium in cutoff strategies is a pair $\left(p^{*}, c^{*}\right)$ such that $p^{*}=F\left(c^{*}\right)$ and either (i) $c^{*}=B_{P}\left(p^{*}\right), c^{*} \in[\underline{c}, \bar{c}]$, or (ii) $c^{*}=\underline{c}>B_{P}(0)$ or (iii) $c^{*}=\bar{c}<B_{P}(1)$. Note that in the definition, we have allowed for corner solutions where agents vote with probability one or zero.

In the case $\lambda<\hat{\lambda}$, it is clear that if the average turnout probability is $p$, and $i$ is type $k=M, D$, then $i^{\prime} s$ (strict) best response is to participate iff $c^{i} \leq B_{k}(p) \equiv \tilde{c}_{k}$. An equilibrium in cutoff strategies is a triple $\left(p^{*}, c_{M}^{*}, c_{D}^{*}\right)$ where $p^{*}=0.5 F\left(c_{M}^{*}\right)+0.5 F\left(c_{D}^{*}\right)$, and either (i) $c_{k}^{*}=B_{k}\left(p^{*}\right), c_{k}^{*} \in[\underline{c}, \bar{c}]$, or (ii) $c_{k}^{*}=\underline{c}>B_{k}\left(p^{*}\right)$, or (iii) $c_{k}^{*}=\bar{c}<B_{k}\left(p^{*}\right)$, for $k=M, D$. Again, $p^{*}$ is the ex ante probability of an agent choosing to vote.

The following proposition demonstrates the existence of equilibria in cutoff strategies, and also proves a characterization:

Proposition 1. If $\lambda>\hat{\lambda}$, there is a unique symmetric Bayesian equilibrium in cutoff strategies. In this equilibrium, all those who turn out, vote according to their private values. If $\lambda \leq \hat{\lambda}$, there is at least one symmetric Bayesian equilibrium in cutoff strategies. In this equilibrium, all those who turnout, vote according to their signals.

When $\lambda>\hat{\lambda}$, existence and uniqueness follows from the fact that that $B_{P}(p)$ is decreasing in $p$, as in Borgers (2004). In the case $\lambda<\hat{\lambda}$, equilibrium existence is guaranteed, but may not be unique. To see why, note that if there is an interior solution for both cutoffs, i.e. $c_{k}^{*}=B_{k}\left(p^{*}\right), k=M, D$, we know from the definition of equilibrium that $p^{*}$ solves

$$
\begin{aligned}
p^{*} & =0.5 F\left(c_{M}^{*}\right)+0.5 F\left(c_{D}^{*}\right) \\
& =0.5 F\left(B_{M}\left(p^{*}\right)\right)+0.5 F\left(B_{D}\left(p^{*}\right)\right) \equiv \Phi_{S}\left(p^{*}\right)
\end{aligned}
$$

Multiple equilibria arise the function $\Phi_{S}(p)$ may be non-decreasing everywhere in $p$, as the following three-voter example shows. 
Example 1 (Multiple Equilibria). Assume $n=3$ and that $c$ is uniform on $[0, \bar{c}]$. The logic of the example is that $n=3$, the probability of being pivotal - and thus $\Phi_{S}(p)$ is high when $p$ is close to zero (since no one votes) or close to one (since both opponents vote, but may have opposite signals), but unlikely when is moderate (since, most likely, only one of the two will vote). In fact, it can be shown that $\Phi_{S}(p)$ is a convex quadratic as shown in Figure 1.

\section{(Figure 1 in here.)}

Then, the voting cost - which is linear in $p$ - may be equal to the benefit at several points, as shown i.e. multiple equilibria.

The details of the computation are as follows. Condition (3.9), plus $F(c)=c / \bar{c}$ from uniform costs, implies

$$
\Phi_{S}(p) \equiv \frac{0.5}{\bar{c}}\left(B_{M}(p)+B_{D}(p)\right)
$$

Moreover, from formulae (3.6), we see that

$$
\begin{aligned}
0.5\left(B_{M}(p)+B_{D}(p)\right)= & (1-\lambda)\left(Q_{+}(p)-Q(p)\right) \\
& (1-\lambda)(q-0.5)\left[2 p^{2} q(1-q)+(1-p)^{2}\right]
\end{aligned}
$$

So, from (3.10):

$$
\Phi_{S}(p)=\frac{1-\lambda}{\bar{c}}(q-0.5)\left[2 p^{2} q(1-q)+(1-p)^{2}\right]
$$

Multiple equilibria can now be demonstrated as follows. Note that $\Phi_{S}(0)=\frac{1-\lambda}{\bar{c}}(q-$ $0.5)>0$, and $\Phi_{S}(1)=\frac{1-\lambda}{\bar{c}}(q-0.5) 2 q(1-q)>0$. So, it is always possible to choose ${ }^{14}$ $\frac{1-\lambda}{\bar{c}}$ so that $\Phi_{S}(p)=p$ has two solutions $p^{*}, p^{* *}$, as shown in Figure 1. In this case, it is obvious that $\Phi_{S}(1)>1$, so that there is a third corner equilibrium, where everybody votes i.e. $p^{* * *}=1$.

But, it should also be noted that multiple equilibria are a possibility only in small electorates. This is because $\Phi_{S}(p) \rightarrow 0$ for any fixed $p$ as $n \rightarrow \infty$, as the probability of being pivotal becomes small. We conjecture ${ }^{15}$ that for $n$ high enough, $p^{*}$ is unique, and then tends to zero as $n \rightarrow \infty$.

\footnotetext{
${ }^{14}$ For example, if we take $q=0.75, \lambda=0$, and $\bar{c}=0.09$, then it is easy to check that the two roots are $p^{*}=\frac{1.3119}{1.375}, p^{* *}=\frac{1.0481}{1.375}$.

${ }^{15}$ Multiplicity is not really the main topic of this paper, so we leave this for future work.
} 


\section{Welfare Analysis}

\subsection{The Turnout Decision}

In this section, we investigate whether equilibrium cutoffs $c^{*}, c_{M}^{*}, c_{D}^{*}$ are efficient. We begin by defining the ex ante payoff to any agent (i.e. prior to observing $t^{i}, \sigma^{i}, c^{i}$ ), conditional on a given cutoff strategy. First, note that if $\lambda \geq \hat{\lambda}$, voting according to private values occurs. In this case, if $i$ does not vote, his payoff is just 0.5 , whatever $\lambda$, as $t^{i}$ is uncorrelated with $t^{j}$ and with $s$. Given an arbitrary cutoff $\hat{c}$, if he does vote, his payoff is $0.5+B_{P}(F(\hat{c}))-c$. So, given an arbitrary cutoff $\hat{c}$, this ex ante payoff is

$$
E W_{P}(\hat{c})=0.5+\int_{\underline{c}}^{\hat{c}}\left(B_{P}(F(\hat{c}))-c\right) f(c) d c
$$

So,

$$
\frac{d E W_{P}(\hat{c})}{d \hat{c}}=\left(B_{P}(F(\hat{c}))-\hat{c}\right)+\int_{\underline{c}}^{\hat{c}} B_{P}^{\prime}(F(\hat{c})) f(\hat{c}) f(c) d c
$$

Now at an interior equilibrium, $B_{P}\left(F\left(c^{*}\right)\right)=c^{*}$, so

$$
\frac{d E W_{P}\left(c^{*}\right)}{d \hat{c}}=\int_{\underline{c}}^{c^{*}} B_{P}^{\prime}\left(F\left(c^{*}\right)\right) f\left(c^{*}\right) f(c) d c<0
$$

where in the inequality, we have used the fact, proved by Borgers (2004), Proposition 1, that $B_{P}(p)$ is decreasing in $p$. So, we have:

Proposition 2. If $\lambda>\hat{\lambda}$, and the unique equilibrium is interior, the equilibrium cutoff $c^{*}$ is locally too high i.e. $\frac{d E W_{P}\left(c^{*}\right)}{d \hat{c}}<0$, and so a small decrease in the cutoff $\hat{c}$ from $c^{*}$ is always ex ante Pareto-improving.

This result generalizes the findings of Borgers' paper to our environment. The intuition is the negative pivot externality: an increase in $\hat{c}$ raises $p$, and thus decreases the probability that any agent is pivotal, and thus their utility.

Now we consider the case if $\lambda \leq \hat{\lambda}$. Here, the key difference is that the payoff to any agent $i$ if he does not vote depends (positively) on the turnout probabilities of others, because the higher $p$, the more likely it is that more signals are used in the decision between the two alternatives. Specifically, it the ex ante payoff to any agent given cutoffs $\left(c_{D}, c_{M}\right)$ is 


$$
\begin{aligned}
E W_{S}\left(c_{D}, c_{M}\right)= & 0.5\left[Q(p)+\int_{0}^{c_{M}}\left(B_{M}(p)-c\right) f(c) d c\right] \\
& +0.5\left[(1-\lambda) Q(p)+\lambda(1-Q(p))+\int_{0}^{c_{D}}\left(B_{D}(p)-c\right) f(c) d c\right] \\
= & 0.5 \lambda+(1-\lambda) Q(p)+0.5\left[\int_{0}^{c_{M}}\left(B_{M}(p)-c\right) f(c) d c+\int_{0}^{c_{D}}\left(B_{D}(p)-c\right) f(c) d c\right]
\end{aligned}
$$

where $p=0.5\left(F\left(c_{M}\right)+F\left(c_{D}\right)\right)$. This says that welfare is the average of ex ante payoffs of $\mathrm{M}$-agents and D-agents. That is, the term in the square brackets in the first line of (4.2) is the ex ante payoff of the M-agent, and the term in the square brackets on the second line is the ex ante payoff of the D-agent. Each is composed of two terms, the baseline payoff to not voting (e.g. $Q(p)$ for the M-agent), and the expected net benefit from voting when the cost is below the cutoff.

Now consider the effect of increasing the cutoff $c_{k}$, starting at an interior equilibrium. Using the envelope conditions, $B_{k}\left(p^{*}\right)=c_{k}^{*}$ which hold at an interior equilibrium, we can write the marginal effect of an increase in cutoff $c_{k}$ on $E W_{S}$ conveniently as follows:

$$
\frac{\partial E W_{S}\left(c_{D}^{*}, c_{M}^{*}\right)}{\partial c_{k}} \frac{1}{0.5 f\left(c_{k}^{*}\right)}=(1-\lambda) Q^{\prime}\left(p^{*}\right)+0.5\left(B_{M}^{\prime}\left(p^{*}\right) F\left(c_{M}^{*}\right)+B_{D}^{\prime}\left(p^{*}\right) F\left(c_{D}^{*}\right)\right)
$$

So, there is an externality in the choice of cutoff that can be analytically decomposed into two parts. The first, measured by $(1-\lambda) Q^{\prime}\left(p^{*}\right)$, is the effect on any agent's expected utility from non-participation of an increase in the cutoff (and thus turnout probability) of others. Thus, $(1-\lambda) Q^{\prime}\left(p^{*}\right)$ measures the information-pooling externality referred to above. We would expect this to be positive and indeed it is, as shown in the proof of Proposition 3 below.

The second, measured by $0.5\left(B_{M}^{\prime}\left(p^{*}\right) F\left(c_{M}^{*}\right)+B_{D}^{\prime}\left(p^{*}\right) F\left(c_{D}^{*}\right)\right)$, is the effect of an increase in $p$ on any agent's benefit from voting. We might call this the pivot externality. Example 1 above indicates that generally, this term can be positive or negative.

The key question is which of these two externalities dominates in determining the sign of $\frac{\partial E W_{S}\left(c_{D}^{*}, c_{M}^{*}\right)}{\partial c_{k}}$. We can show that in the event that the pivot externality is negative, it is dominated by the positive information externality:

Proposition 3. Assume $\lambda<\hat{\lambda}$. Then, at any interior equilibrium, both equilibrium cutoffs are too low i.e. $\frac{d E W_{S}\left(c_{D}^{*}, c_{M}^{*}\right)}{d c_{k}}>0, k=M, D$ and so a small increase in the cutoff $c_{k}$ from $c_{k}^{*}$ is always ex ante Pareto-improving. 
Proposition 3 contrasts sharply with Borgers' results. Of course, in the pure common values case i.e. when $\lambda=0$, our result is not surprising at all. But it is quite striking that there can be too little turnout irrespective of the precise value of $\lambda$; what determines the direction of inefficiency is not the bias per se, but how individuals decide to vote if they do turn out.

It also suggests that if there are multiple voting equilibria, the one with higher turnout might be more efficient. Unfortunately, such a "global" result is generally not true, for the following reason. To keep matters simple, we consider the pure common values case when $\lambda=0$. In this case note that $B_{M}(p)=B_{D}(p)=Q_{+}(p)-Q(p)$ and $c_{M}^{*}=c_{D}^{*}=c^{*}$. For economy of notation, let $Q_{+}(p)-Q(p) \equiv B(p)$ just in this case. Then, the expression for $E W_{S}$ i.e. (4.2), simplified to the pure common values case, is

$$
E W_{S}\left(c^{*}\right)=Q\left(F\left(c^{*}\right)\right)+\int_{0}^{c^{*}}\left(B\left(F\left(c^{*}\right)\right)-c\right) f(c) d c
$$

Consider two symmetric voting equilibria with cutoffs $c^{*}$ and $c^{* *}$ such that $c^{*}<c^{* *}$. Using (??), and integrating by parts, the difference between the expected payoffs at the two equilibria can be written as

$$
E W_{S}\left(c^{* *}\right)-E W_{S}\left(c^{*}\right)=\int_{c^{*}}^{c^{* *}}\left(Q^{\prime}(F(c))+F(c) B^{\prime}(F(c))\right) f(c) d c+\int_{c^{*}}^{c^{* *}}(B(F(c))-c) f(c) d c
$$

By the argument that proves Proposition 3, we know that the first integral is positive. However, the sign of the second integral is ambiguous as $B(p)-c$ is, in general, nonmonotonic. This makes it impossible to obtain a general Pareto-ranking of equilibria. In particular, we cannot show that, in general, a Bayesian equilibrium with a higher cutoff value Pareto-dominates a Bayesian equilibrium with a lower cutoff value.

However, the following result shows that with multiple equilibria, it is possible to find an equilibrium with higher turnout that gives higher ex ante welfare than one with lower turnout.

Proposition 4. Assume that $\lambda=0$ so that $c_{D}=c_{M}=c^{*}$. Suppose that there are $m$ voting equilibria as represented by cutoffs: $c^{*}=c_{i}, c_{1}<. .<c_{k}<\ldots<c_{m}$. (i) If either (a) $m \geq 2$, and $B(1)<\bar{c}$ or (b) $m \geq 3$, there is some $k, 1 \leq k \leq m-1$, such that the the voting equilibrium $c_{k+1}$ Pareto dominates the voting equilibrium $c_{k}$. (ii) If $B(1) \geq \bar{c}$, then compulsory voting is an equilibrium i.e. $c_{m}=\bar{c}$, and Pareto-dominates the equilibrium $c_{m-1}$.

Note that part (ii) of the above result is of limited interest with a large potential electorate; the inequality $B(1) \geq \bar{c}$ is unlikely to be satisfied if $n$ is large, as $\lim _{n \rightarrow \infty} B(1)=$ 
0. Moreover, as already discussed, multiple equilibria may become less likely as $n$ becomes large. So, perhaps Proposition 4 is more applicable to small electorates e.g. committees.

\subsection{Compulsory vs. Voluntary Voting}

The striking result of Borgers' paper is that in the case of private values, voluntary voting always dominates compulsory voting. That result is the global analog of the local inefficiency result stated in Proposition 3 above. By an application of the arguments in Borgers' paper, in particular the proof of Proposition 2, it is possible to show that when $\lambda>\hat{\lambda}$, compulsory voting is always undesirable. That is, Borgers' result generalizes to the case when there are also a common element to payoffs, as long as these are sufficiently unimportant so that voters vote according to their private values.

What about the case where agents vote according to their signals? The positive externality in this case suggests that there should be some conditions under which compulsory majority voting Pareto dominates Bayesian equilibrium outcomes with voluntary majority voting. One such condition, which is of particular interest for small electorates, is in fact clear from Proposition 4: i.e. $B(1) \geq \bar{c}$, starting at equilibrium $c_{m-1}$, imposing compulsory voting moves everybody to equilibrium $\bar{c}$ and is thus Pareto-improving.

Can compulsory voting lead to a Pareto-improvement even when $\bar{c}$ is not a voting equilibrium threshold? The following example shows that this a robust possibility. In this example, there is a unique equilibrium with $\hat{c}<\bar{c}$, and starting at this equilibrium, imposing compulsory voting leads to a strict Pareto-improvement.

\section{Example 2 (Compulsory Voting May Be Desirable).}

The Example is the same as Example 1 with the additional assumption that $\lambda=0$. Ex ante payoffs can thus be computed from formula (4.4), using $f(c)=\frac{1}{\bar{c}}$ and $c_{M}=c_{D}=c^{*}$, $p=c^{*} / \bar{c}$, which gives $E W_{S}$ just as a function of $p$;

$$
\begin{aligned}
E W_{S}(p) & =Q(p)+\frac{1}{\bar{c}} \int_{0}^{p \bar{c}}(B(p)-c) d c \\
& =Q(p)+p B(p)-\bar{c} p^{2} / 2
\end{aligned}
$$

for any voting probability $p$. Moreover, from the Appendix, we have

$$
\begin{aligned}
& Q(p)=0.5(1-p)^{2}+2 p(1-p) q+p^{2} q \\
& B(p)=(q-0.5)\left[2 p^{2} q(1-q)+(1-p)^{2}\right]
\end{aligned}
$$

So, substituting (4.6) into (4.5), we get

$$
E W_{S}(p)=0.5(1-p)^{2}+2 p(1-p) q+p^{2} q+p(q-0.5)\left[2 p^{2} q(1-q)+(1-p)^{2}\right]-\bar{c} p^{2} / 2
$$


Now from the discussion of Figure 1, it is clear that for $\bar{c}$ high enough, there is a unique interior equilibrium $p^{*}$. Generally, the condition required is that

$$
\bar{c}>B(1)=Q_{+}(1)-Q(1) \equiv \psi
$$

Now choose $q=0.75$. Then from (4.6), $\psi=0.09375$. So, take $\bar{c}=0.0938$. Then, by construction, $p^{*}$ is the unique root in $(0,1)$ to $p \bar{c}=B(p)$, which, given the values already chosen for $q$ and $\bar{c}$, can be calculated as $p^{*}=0.72689$. So, to show that compulsory voting voting Pareto-dominates the equilibrium, it is enough to show that $E W_{S}(1)>$ $E W_{S}\left(p^{*}\right)$. But, using the general formula (4.7), we can compute that $E W_{S}(1)=0.79685>$ $0.75613=E W_{S}\left(p^{*}\right)$.

\section{Comparative Statics: The Effects of Changing Bias and Infor- mation Quality}

\subsection{Personal Bias and Equilibrium Turnout}

How does the equilibrium turnout probability $p^{*}$ change as personal bias - as measured by $\lambda$ - increases? In the "regime" where $\lambda>\hat{\lambda}$, i.e. voters vote according to private values, $p^{*}$ is determined by $p^{*}=F\left(B_{P}\left(p^{*}\right)\right)$. From Lemma $2, B_{P}(p)$ is increasing in $\lambda$, and decreasing in $p$. So, the equilibrium $p^{*}$ is increasing in $\lambda$. This is intuitive; the higher $\lambda$, the greater the benefit from voting according to private values.

A reverse argument applies "regime" where $\lambda>\hat{\lambda}$, i.e. voters vote according to signals. From Lemma $3, B_{D}(p)$ is decreasing in $\lambda$. But now, there is the complication of multiple equilibria. If equilibrium is unique, i.e. $\Phi_{S}(p)$ is downward-sloping everywhere, and as it is also decreasing in $\lambda, p^{*}$ will be decreasing in $\lambda$. But, if there are multiple equilibria, as in Example 1 above, the intermediate equilibrium $p^{* *}$ in Figure 1 will increase with $\lambda$.

A final question concerns the continuity of $p^{*}$ as a function of $\lambda$ at $\lambda=\hat{\lambda}$. At this point, there are two possible equilibrium turnout probabilities, $p_{S}$ and $p_{P V}$. At this point, there are two forces which tend to make $p_{S}<p_{P V}$.

First, as signals are correlated, there is an incentive to "free-ride" if others are voting according to signals; from the perspective of some agent $i$, any other agent will vote "correctly" with probability $q>0.5$, as compared to 0.5 when agents ore voting according to private values. Second, the benefit from one's most preferred alternative relative to random selection is $q-0.5<0.5$ in the signals regime, but 0.5 in the private values regime, as then voters are sure which alternative is best. 
All these general arguments can be made precise in the two-person case. In particular, we have:

Proposition 5. If $n=2$, and $c$ is uniform on $[0,1], p^{*}$ is unique. Also, $p^{*}$ as a function of $\lambda$ is as follows: for $\lambda \leq \hat{\lambda}, p^{*}=p_{S}=\frac{(1-\lambda)(q-0.5)}{1+(1-\lambda)(q-0.5)}$, and for $\lambda \geq \hat{\lambda}, p^{*}=p_{P V}=\frac{0.5 \lambda}{1+0.25 \lambda}$. At $\hat{\lambda}, p_{S}<p_{P V}$.

This Proposition can be illustrated using the following diagram. Using the formulae in Example 2, plus $\hat{\lambda}=(q-0.5) / q$, it is simple to calculate that at $\lambda=\hat{\lambda}, 0<p_{S}<p_{P V}$. So, as already argued, there $p^{*}$ as a function of $\lambda$ initially falls to a minimum at $\hat{\lambda}$, and then there is there is a discontinuous jump upward at $\hat{\lambda}$ from $p_{S}$ to $p_{P V}$. This is illustrated in Figure 2 below.

(Figure 2 in here.)

\subsection{The Effects of Better Information}

In this section, we consider the positive and normative effects of a change in the accuracy of the signal, $q$. First, consider the effects on turnout behavior. Most obviously, an increase in $q$ causes the critical weight $\hat{\lambda}$ to rise, so that voting may switch from private values to signals. Call this a regime switch. What about the effect on turnout? If there is no regime switch effect, then obviously, if $\lambda>\hat{\lambda}$, there is no effect at all on the equilibrium.

If $\lambda<\hat{\lambda}$, then we know that the aggregate turnout probability is determined by $Q_{+}(p)-Q(p)$. Now, at fixed $p$, this need not be an increasing function of $q$ (see (3.8)). Even if it is increasing in $q, p^{*}$ need not be when there are multiple equilibria. For example, in Figure 1, if $\Phi_{S}(p)$ shifts up with $q, p^{* *}$ will fall. Thus, the behavior of $p^{*}$ with respect to $q$ may be quite complex.

It is easier to study how the turnout probability $p^{*}$ varies with $q$ in the two-agent case, where we already know that $p^{*}$ is unique from Proposition 5. In this case, a increase in $q$ can cause a "regime switch" from voting according to private values to voting according to signals, and this can cause a fall in $p^{*}$. This is clear from figure 2, which illustrates the equilibrium turnout probabilities as a function of $\lambda$ for the case of two agents and costs distributed on $[0,1]$. Assume that initially, $\lambda=\frac{(q-0.5)}{q}+\varepsilon$, for $\varepsilon$ small, so that voting is according to private values. Then, the equilibrium turnout probability is just to the right of at point $A$ in Figure 2. Now suppose $q$ increases slightly to $q^{\prime}$, so that there is a regime switch i.e. $\lambda=\frac{(q-0.5)}{q}+\varepsilon<\frac{\left(q^{\prime}-0.5\right)}{q^{\prime}}$. Then, on Figure 2, the equilibrium turnout probability will switch ${ }^{16}$ to a value close to the left of $B$ on Figure 2. So, as long as the

\footnotetext{
${ }^{16}$ More precisely, two things will happen. First, the segment of $p^{*}$ when $\lambda<\hat{\lambda}$ will shift up slightly.
} 
increase in $q$ is small, the turnout probability can fall. So, increased information can lead to a fall in turnout probabilities.

This point is now made more rigorously via a numerical example, illustrated in the following Table. We set $\lambda=1 / 3$. This implies that $\lambda=\hat{\lambda}$ at $q=0.75$. So, at $q=0.75$, there are two possible equilibrium values of $p^{*}, p_{S}$ and $p_{P V}$. For $q>0.75$, voting is according to signals, i.e. $p^{*}=p_{S}$, as indicated in the Table. Similarly, for $q<0.75$, voting is according to private values, i.e. $p^{*}=p_{P V}=0.154$, also as indicated. So, if we select the "signals" equilibrium at $q=0.75$, we can see that $p^{*}$ drops discontinuously with $q$ at $q=0.75$, from 0.154 to 0.143 and remains below $p_{P V}=0.154$ until $q=0.78$. As $q$ continues to rise, $p^{*}$ eventually rises above $p_{P V}=0.154$.

\section{Table 1 in here}

In this example, we are also able to calculate the effect on welfare of an increase in $q$ through the critical value of $q=0.75$ where the "regime switch" occurs. The appropriate criterion is ex ante welfare evaluated at the beginning of stage 0 i.e. before agents observe their preferences or information, as given by formulae (4.1), (4.2). In this case, using the properties of the uniform distribution, and $n=2$, we get $^{17}$ the following simplified formulae for $E W_{P}$ of ;

$$
E W_{P}=0.5+0.5\left(p^{*}\right)^{2}
$$

and also for $E W_{S}$;

$$
E W_{S}=0.5+(1-\lambda)(q-0.5) p^{*}+0.25\left[\left(c_{M}^{*}\right)^{2}+\left(c_{D}^{*}\right)^{2}\right]
$$

Finally, $p^{*}$ is given by the formulae in Proposition 5 , and $c_{M}^{*}=B_{M}\left(p^{*}\right)=(q-0.5)(1-$ $\left.p^{*}\right), c_{D}^{*}=B_{D}\left(p^{*}\right)=(1-2 \lambda)(q-0.5)\left(1-p^{*}\right)$. Combining these formulae with (5.1), (5.2), we can calculate $E W_{S}, E W_{P}$ and these are shown in the last two lines of Table 1. As can be seen, welfare rises monotonically with $q$, even though $p^{*}$ jumps downward at $q=0.75$. Indeed, at $q=0.75$, where there are two equilibria, the one where voting according to private values has lower welfare even though the level of participation is higher. This of course, is only one example. However, extensive experimentation with this example by changing the value of $\lambda$ reveals that expected welfare is invariably increasing in $q$, even when there is a regime switch.

Second, the equilibrium turnout probability will switch onto this segment.

${ }^{17}$ From (4.1), using the facts that $c=p, f(c)=1, E W_{P}=0.5+p B_{P}(p)-p^{2} / 2$. Then, from the equilibrium condition $p=B_{P}(p), E W_{P}=0.5+p^{2}-p^{2} / 2=0.5+0.5 p^{2}$. The derivation of a simplified formula for $E W_{S}$ follows similar lines. 


\section{Related Literature and Conclusions}

\subsection{Extensions}

So far, we have assumed that information is made available costlessly. Now, suppose that the cost of information is strictly positive i.e. $k>0$, and at stage 0 , agents can decide whether or not to purchase the signal. First, it is clear that if $\lambda>\hat{\lambda}$, no information is ever purchased. This is by Lemma 1: if an agent purchased such information, he would never use it at the voting stage, and so it would be worthless. The picture when $\lambda<\hat{\lambda}$ is less clear. It can be shown (details on request) that under fairly mild conditions, there are two strictly positive numbers $\underline{V}<\bar{V}$ such that if $k<\underline{V}$, all agents buy information in equilibrium, and $k>\bar{V}$, no agents buy information in equilibrium. The interpretation of these numbers is that $\bar{V}$ (resp. $\underline{V}$ ) is the value of information to an agent at the voting stage if he rationally anticipates that no other agents (all other agents) will purchase information at stage 0 .

Perhaps a more serious limitation is that the degree of bias is the same for all agents. However, we believe that relaxing this would make the model very difficult to analyze (indeed, intractable for $n>2$ ) while not really changing the flavour of the results. This is certainly a topic for future work.

\subsection{Related Literature}

Other than Borgers (2004), there are a number of papers related to our work. First, two other papers question the robustness of Borger's striking result on excessive participation when voting is costly. Goree and Grosser (2004) relax Borgers' assumptions by allowing preferences over alternatives to be positively correlated, and show that if there is sufficient positive correlation, there is too little voting in equilibrium. Krasa and Polborn (2006) allow the probability that a voter prefers a particular alternative (say $A$ ) to be different from 0.5, relaxing symmetry in Borgers' model, and shows that when the number of agents is large enough, the voting externality is generically positive, rather than negative; intuitively, voters in the majority vote too little, hoping to free-ride on others in their group who do vote. Taylor and Yildirim(2005) push this argument further. Their baseline model is the same as Krasa and Polborn's, except that the cost of voting is not random, but fixed at some value that is common knowledge. Then, free-riding by the majority group exactly offsets their numerical advantage, so that in equilibrium, the probability that the majority's preferred option is chosen is exactly one-half, an inefficient outcome. They show that if voters are uncertain about the probability that other voters prefer 
alternative $A$, this free-riding effect is diminished, leading to a welfare improvement. In this case, voter turnout is also lower.

Our paper has similar findings, but has a different story behind them. First, we find that even with a fixed number of voters, the voting externality can be positive, but the reason is that when voter bias is relatively low, voters are basing their decisions on the information they get, not their private preferences. Second, we find that better information can lower voter turnout. But this is due to better information casing voters to "switch regime" in their voting behavior.

There is also a related literature ${ }^{18}$ on endogenous information acquisition (Mukhopadhaya (2003), Persico (2000), Martinelli (2006)) in a setting where agents have identical preferences over alternatives depending on an unknown state of the world, but can buy costly signals on the state of the world. This literature focuses on the free rider problem in information acquisition. Unlike us, this literature does not allow for endogenous, costly turnout: informed voters - those who have bought the signal - are assumed to vote with probability 1 . The focus of this literature is on the probability with which agents acquire information, rather than on the probability of turnout. But, the special case of our model where there are no private values $(\lambda=0)$ can be interpreted as a model of this type, because no agent would acquire information and not vote subsequently, meaning that our turnout cost $c$ can also be interpreted as a cost of information acquisition. So, some of our results, notably Proposition 4 and Example 1, can be interpreted as contributions to this literature. In particular, while Mukhopadhaya (2003) points out that there can be a "free-rider" problem in information acquisition, it has not previously been recognized that this can give rise to multiple equilibria, which can be partially Pareto-ranked.

\subsection{Conclusion}

In this paper, we have shown that in a model of costly voting where preferences are a convex combination of a private values component and a common values component, when the weight on the common values component is sufficiently high, the nature of the inefficiency of voting equilibrium identified in Borgers (2004) is reversed: even with heterogenous preferences, in the vicinity of a Bayesian equilibrium, higher turnout is always

\footnotetext{
${ }^{18}$ One variant of this literature, initiated by the important paper of Persico(2004), is on the optimal design of electoral rules when aquisition of information is endogenous. Here, the statistically optimal decision rule, given reported signals by all the decision-makers (which in our set-up is simply "majority rule" i.e. to choose the alternative indicated by the majority of reported signals) might not give the best ex ante incentives to acquire information (Gerardi and Yariv(2005)).
} 
Pareto-improving. In addition, we have also shown that Pareto ranked turnout equilibria may exist and moreover, compulsory majority voting can Pareto dominate voluntary majority voting. The key behind all the results in this paper lies in the finding that there are two different externalities at work: the negative "pivot" externality identified by Borgers (2004) and the positive information externality. In the vicinity of a Bayesian equilibrium, the positive informational externality may outweigh the negative "pivot" externality implying that there is too little turnout in the voting process. We show that an increase in the precision of the signal may lead to fall in turnout with adverse welfare consequences and that when $\lambda<\hat{\lambda}$, the value of information (as determined in equilibrium) is strictly positive and therefore, when the cost of purchase of the signal is low enough, there will be costly signal purchase for a whole range of $\lambda^{\prime} s$. 


\section{References}

[1] Austen-Smith, D. and J.S. Banks (1996), "Information Aggregation, Rationality and the Condorcet jury theorem", American Political Science Review, 90, pp.34-45.

[2] Borgers, T. (2004), "Costly Voting”, American Economic Review, 94(1), pp.57-66.

[3] Duggan. J. and C. Martinelli (2001), "A Bayesian Theory of Voting in Juries", Games and Economic Behavior, 37, 259-294

[4] Gerardi,D and L. Yariv(2005), "Information Acquisition in Committees", unpublished paper, Yale University

[5] Goeree, J.K. and J. Grosser (2004), "False consensus voting and welfare reducing polls", mimeo, California Institute of Technology.

[6] Groseclose, T. (2001), "A Model of Candidate Location When One Candidate Has a Valence Advantage", The American Political Science Review, 95(4), pp.862-886.

[7] Feddersen, T and W. Pessendorfer (1997), "Voting Behavior and Information Aggregation in Elections with Private Information", Econometrica, 65(5), pp.1029-1058.

[8] Feddersen, T and W. Pessendorfer (1999), "Abstention in Elections with Asymmetric Information and Diverse Preferences", The American Political Science Review, 93(2), pp.381-398.

[9] Groseclose, Tim, (2001) "A Model of Candidate Location When One Candidate Has a Valence Advantage," American Journal of Political Science, 45, 862-886.

[10] Krasa, S. and M. Polborn (2006), "Is mandatory voting better than voluntary voting?", mimeo.

[11] McLennan, A. (1998), "Consequences of the Condorcet Jury Theorem for Beneficial Information Aggregation by Rational Agents", American Political Science Review, $92,413-418$

[12] Matsusaka, J. G. (1995), "Explaining Voter Turnout Patterns: An Information Theory", Public Choice v84, n1-2 : 91-11\%.

[13] Mukhopadhaya, K. (2003), "Jury size and the free rider problem", Journal of Law, Economics and Organization, 19, pp. 24-44. 
[14] Persico, N. (2004), "Committee design with endogenous information", Review of Economic Studies, 71, 165-191.

[15] Rothschild, M. and Stiglitz, J. (1970), "Increasing Risk I: A definition", Journal of Economic Theory, 2, pp.225-243.

[16] Taylor, C., Yildirim, H. (2005), "Public information and electoral bias", Duke University, Department of Economics, Working Papers: 05-11. 


\section{A. Appendix}

Proof of Lemma 1. (i) If there are $l$ other voters, voter $i$ is pivotal only in two possible cases. Case 1 is when $l$ is even and $\frac{l}{2}$ voters vote for $A$ while the other $\frac{l}{2}$ voters vote for $B$. Case 2 is when $l$ odd and $\frac{l+1}{2}$ voters have voted for $A$, and $\frac{l-1}{2}$ for $B$, or vice versa. So, in general, to demonstrate an equilibrium, we only need to show that the relevant voting strategy played by $i$ is a best response to the same voting strategy played by the other $l$ voters in each of these two cases.

(ii) Assume all other $j \neq i$ vote with their private values. W.l.o.g, assume that Case 1 applies (the argument is the same in Case 2). Also, by definition, the interesting case is where $t^{i} \neq \sigma^{i}$. Assume she votes for alternative $t^{i}$. As she is pivotal, the outcome is $t^{i}$, which gives her a payoff $\lambda+(1-\lambda) .(1-q)$. This is because the private value part of her payoff is 1 , but the common value part is $1-q$, as she is voting "against" her signal and thus evaluates the probability that $t^{i}=s$ at $1-q$.

Alternatively, assume she votes for alternative $\sigma^{i}$. As she is pivotal, the outcome is $\sigma^{i}$, which gives her a payoff $(1-\lambda) . q$. This is because the private value part of her payoff is 0 , but the common value part is $q$, as she is voting "with" her signal and thus evaluates the probability that $t^{i}=s$ at $q$.

So, she prefers to vote with her private value if $\lambda+(1-\lambda) .(1-q) \geq(1-\lambda) . q$, or $\lambda \geq(q-0.5) / q=\hat{\lambda}$.

(iii) Assume all other $j \neq i$ vote with their signals. Assume also Case 1 i.e. $l$ is even and $\frac{l}{2}$ voters vote for $A$ while the other $\frac{l}{2}$ voters vote for $B$. Conditional on this event, $i$ knows that $\frac{l}{2}+1$ " $A$ " signals, and $\frac{l}{2}$ " $B$ " signals have been observed. So, he believes that state of the world $s_{A}$ has occurred with probability $q$. So, the payoffs to voting with her private value or signal are just as calculated in part (ii) above. So, he prefers to vote according to her signal if $\lambda \leq \hat{\lambda}$.

Now consider Case 2. This is more difficult. Assume w.l.o.g. that $\sigma^{i}=A$. We distinguish two subcases. Case $2(\mathrm{i})$ is where $\frac{l+1}{2}$ voters have voted for $A$, and $\frac{l-1}{2}$ for $B$. In this case, $i$ infers if he is pivotal, that there are two more " $A$ " signals than " $B$ " signals. So, he calculates ${ }^{19}$ the probability that $\sigma^{i}=s$ at $\chi=\frac{q^{2}}{q^{2}+(1-q)^{2}}$. So, the expected payoff to voting according to signal and private value are thus $\chi(1-\lambda)$ and $\lambda+(1-\chi)(1-\lambda)$ respectively.

Case 2(ii) is where $\frac{l+1}{2}$ voters have voted for $B$, and $\frac{l-1}{2}$ for $A$. In this case, $i$ infers

\footnotetext{
${ }^{19}$ Formally, $\chi$ is equal to the posterior probability that the state is (say) $A$, given that there are $l+1$ (resp. $l-1$ ) signals in favour of $A$ (resp. B). Using Bayes' rule, after some simplification, we get the formula in the text.
} 
that if he is pivotal, there are equal numbers of " $A$ " and " $B$ " signals. So, he calculates the probability that $\sigma^{i}=s$ at just 0.5 . So, the expected payoff to voting according to signal and private value are thus $0.5(1-\lambda)$ and $\lambda+0.5(1-\lambda)$ respectively.

Conditional on $l$ odd, $i$ does not know which of Cases 2(i) and 2(ii) has occurred when he decides whether to vote with his signal or his private value. But, he calculates as follows. Let $l_{A}$ the number of other voters who receive signal $\sigma^{j}=A$. Then the probability of case $2(\mathrm{i})$ is

$$
\begin{aligned}
\operatorname{Pr}\left(l_{A}\right. & \left.=\frac{l+1}{2} \mid \sigma^{i}=A\right)=\sum_{k=A, B} \operatorname{Pr}\left(l_{A}=\frac{l+1}{2} \mid s=s_{k}\right) \operatorname{Pr}\left(s=s_{k} \mid \sigma^{i}=A\right) \\
& =g\left(\frac{l+1}{2}: l, q\right) q+g\left(\frac{l+1}{2}: l, 1-q\right)(1-q) \\
& =\left(q^{2}+(1-q)^{2}\right) K
\end{aligned}
$$

using the Binomial formula, where $K=C_{l}^{(l+1) / 2}(q \cdot(1-q))^{(l-1) / 2}$. And similarly, the probability of case 2 (ii) is

$$
\begin{aligned}
\operatorname{Pr}\left(l_{A}\right. & \left.=\frac{l-1}{2} \mid \sigma^{i}=A\right)=g\left(\frac{l-1}{2}: l, q\right) q+g\left(\frac{l-1}{2}: l, 1-q\right)(1-q) \\
& =2 q(1-q) K
\end{aligned}
$$

So, the relative probability of case $2(\mathrm{i})$ is

$$
\eta=\frac{q^{2}+(1-q)^{2}}{q^{2}+(1-q)^{2}+2 q(1-q)}=q^{2}+(1-q)^{2}
$$

So, the overall expected gain to $i$ from voting according to his signal, rather than for his personal preference, is

$$
\begin{aligned}
\Delta & =\eta[(1-\lambda) \chi-\lambda-(1-\lambda) \chi]+(1-\eta)[0.5(1-\lambda)-\lambda-(1-\lambda) 0.5] \\
& =(1-\lambda)(2 q-1)-\lambda
\end{aligned}
$$

So, $\Delta \geq 0$ if $\lambda \leq \hat{\lambda}$.

(iv) Fix a value of $l$, the number of other voters have chosen to participate. For any voter $i$, if both his private value and his signal agree, he is indifferent about voting according to either his private value or signal and moreover, either of the two preceding choices weakly dominates all other pure strategies. When the private value component and the signal disagree, the computations reported in (i) and (ii), taken together, imply that when $\lambda \leq \hat{\lambda}$, voting according to the signal weakly dominates all other pure strategies 
while if $\lambda \geq \hat{\lambda}$, voting according to the private value weakly dominates all other pure strategies.

Proof of Lemma 2. In this case all agents rationally anticipate that voting according to private values will take place in the voting subgame. The expected gain to voting is the probability that $i$ is pivotal, times the expected benefit to voting, given that he is pivotal. There are then two subcases.

(a) $l$ even. In this case, for $i$ to be pivotal, $l / 2$ other voters vote for each of $A$ and $B$ according to their private preference, so this event occurs with probability $g\left(\frac{l}{2}: l, 0.5\right)$. So, the expected payoff to not voting for $i$ is 0.5 . If $i$ votes, he gets alternative $t^{i}$ with probability 1 , which gives him an expected payoff of $(1-\lambda) 0.5+\lambda$. The difference is $\frac{\lambda}{2}$.

(b) $l$ odd. In this case, for $i$ to be pivotal, $(l+1) / 2$ other voters vote for $A$, and $(l-1) / 2$ other voters vote for $B$. If $t^{i}=A$, the gain to voting is zero as the outcome is not affected. If $t^{i}=B$, then the payoff to voting is 0.5 (as the two alternatives tie), and the payoff to not voting is $(1-\lambda) 0.5$ (as $A$ is chosen with probability 1 ). Again, the gain to voting is $\lambda 0.5$, and the probability of this event is $g\left(\frac{l-1}{2}: l, 0.5\right)$.

Proof of Lemma 3. By assumption, $\lambda<\hat{\lambda}$, so voters vote following signals. Suppose exactly $l$ other agents decide to vote. Then, the payoffs to non-participation and participation respectively for the $M$-agent are $Q(l)$ and $Q(l+1)$, because for the $M$-agent, an alternative that matches the state is also the best from for private preferences. So, $b_{M}(l)=Q(l+1)-Q(l)$.

But, the payoffs to non-voting and voting respectively for the $D$-agent are $(1-\lambda) Q(l)+$ $\lambda(1-Q(l))$ and $(1-\lambda) Q(l+1)+\lambda(1-Q(l+1))$, because for the $D$-agent, an alternative that matches the state is the worst for private preferences. So, $b_{D}(l)=(1-2 \lambda)(Q(l+$ 1) $-Q(l))$.

Formulae for $Q_{+}(p)-Q(p)$. First, from (3.6),

$$
\begin{aligned}
& Q(0)=0.5, Q(1)=q, Q(2)=q^{2}+2 q(1-q) \cdot 0.5=q, \\
& Q(3)=q^{3}+3 q^{2}(1-q)=3 q^{2}-2 q^{3} .
\end{aligned}
$$

So, in the case $n=2$,

$$
Q(p)=(1-p) 0.5+p q, Q_{+}(p)=(1-p) q+p q=q
$$

and so

$$
Q_{+}(p)-Q(p)=(q-0.5)(1-p)
$$

In the case $n=3$, a similar calculation gives

$$
\begin{aligned}
Q(p) & =(1-p)^{2} 0.5+2 p(1-p) q+p^{2} q \\
Q_{+}(p) & =(1-p)^{2} q+2 p(1-p) q+p^{2}\left(3 q^{2}-2 q^{3}\right)
\end{aligned}
$$


Then,

$$
\begin{aligned}
Q_{+}(p)-Q(p) & =(1-p)^{2}(q-0.5)+p^{2} q\left(3 q-2 q^{2}-1\right) \\
& =(q-0.5)\left[2 p^{2} q(1-q)+(1-p)^{2}\right]
\end{aligned}
$$

Proof of Proposition 1. If $\lambda \geq \hat{\lambda}$ the continuation voting equilibrium is private values voting, so existence and uniqueness follows directly from Proposition 1 of Borgers (2004) which, in turn, follows from the fact that $B_{P}(p)$ is decreasing in $p$.

Assume now $\lambda<\hat{\lambda}$, so that voting is according to signals. Define the map $f:[0,1] \rightarrow$ $[0,1]$ as follows:

$$
f(p)=0.5 F\left(\tilde{B}_{M}(p)\right)+0.5 F\left(\tilde{B}_{D}(p)\right)
$$

where for each $k=M, D$

$$
\tilde{B}_{k}(p)=\left\{\begin{array}{c}
B_{k}(p), \text { if } B_{k}(p) \in[\underline{c}, \bar{c}] \\
\underline{c}, \text { if } \underline{c}>B_{k}(p) \\
\bar{c}, \text { if } \bar{c}<B_{k}(p)
\end{array}\right.
$$

As $B_{k}(p)$ is continuous in $p, \tilde{B}_{k}(p)$ is also continuous in $p, k=M, D$. As $F$ is continuous, $f(p)$ is continuous and has a fix point $p^{*}$ which corresponds to a turnout equilibrium.

Proof of Proposition 3. From (3.6), note that

$$
B_{M}^{\prime}(p)=Q_{+}^{\prime}(p)-Q^{\prime}(p), B_{D}^{\prime}(p)=(1-2 \lambda)\left(Q_{+}^{\prime}(p)-Q^{\prime}(p)\right)
$$

So, we can write

$$
\begin{aligned}
\frac{\partial E W_{S}\left(c_{D}^{*}, c_{M}^{*}\right)}{\partial c_{k}} & \propto(1-\lambda) Q^{\prime}\left(p^{*}\right)+0.5\left(F\left(c_{M}^{*}\right)+(1-2 \lambda) F\left(c_{D}^{*}\right)\right)\left(Q_{+}^{\prime}(p)-Q^{\prime}(p)\right) \\
& =(1-\lambda-A) Q^{\prime}\left(p^{*}\right)+A Q_{+}^{\prime}\left(p^{*}\right), A=0.5\left(F\left(c_{M}^{*}\right)+(1-2 \lambda) F\left(c_{D}^{*}\right)\right)
\end{aligned}
$$

Now, as $\hat{\lambda}=\frac{q-0.5}{q}<0.5,1-\lambda>0.5$. Also, by definition, $0 \leq A<0.5$. So, at least one of the weights on $Q^{\prime}\left(p^{*}\right), Q_{+}^{\prime}\left(p^{*}\right)$ in the above formula are strictly positive. So, it suffices to show that $Q^{\prime}(p), Q_{+}^{\prime}(p)>0$. First,

$$
Q\left(p^{\prime}\right)-Q(p)=\sum_{m=0}^{n-1}\left(g\left(l: n-1, p^{\prime}\right)-g(l: n-1, p)\right) Q(l) .
$$

Now, for $p^{\prime}>p,\left\{g\left(l: n-1, p^{\prime}\right)\right\}_{l=0}^{n-1}$ first-order stochastically dominates $\{g(l: n-1, p)\}_{l=0}^{n-1}$. Moreover, It is well-known that $Q(l)$ is monotonically increasing in $l$. So, from Rothschild and Stiglitz(1970), we have $Q\left(p^{\prime}\right) \geq Q(p)$. As $Q$ is a polynomial in $Q$, is is differentiable, and the result follows. The same argument applies to show that $Q_{+}^{\prime}(p)>0$. 
Proof of Proposition 4. (i) Define $B(p)=Q_{+}(p)-Q(p)$ as in the text. As $B(1)<\bar{c}$ remark that at $p=F\left(c_{m}\right), B^{\prime}(p)<0$. As $m \geq 2$, it follows that there is at least one Bayesian equilibrium with cutoff $c_{k}$, for some $k, 1 \leq k \leq m-1$ so that $B^{\prime}(p)>0$, $p=F\left(c_{k}\right)$ for some $k<m$. As $B^{\prime}(p)>0, p=F\left(c_{k}\right)$, for some $k<m, B(F(c))>c$, $c \in\left(c_{k}, c_{k+1}\right)$. Alternatively, suppose there exist at least three voting equilibria. Then, there is at least one voting equilibrium with cutoff $c_{k}$ so that $B^{\prime}(p) \geq 0, p=F\left(c_{k}\right)$ for some $k<m$. As, by assumption at $c_{k}, B^{\prime}(p) \geq 0, p=F\left(c_{k}\right)$, for some $k<m, B(F(c)) \geq c$, $c \in\left(c_{k}, c_{k+1}\right)$. So, in both cases, from (4.6), $E W_{S}\left(c_{k+1}\right)>E W_{S}\left(c_{k}\right)$ i.e. the voting equilibrium with the cutoff $c_{k+1}$ Pareto dominates the voting equilibrium with cutoff $c_{k}$. (ii) Next, given that $B(1) \geq \bar{c}, c_{m}=\bar{c}$ follows directly from Proposition 1. By definition of $c_{m}, c_{m-1}, B(F(c)) \geq c, c \in\left(c_{m-1}, c_{m}\right)$. So, from (4.6), $E W_{S}(\bar{c})=E W_{S}\left(c_{m}\right)>E W_{S}\left(c_{m-1}\right)$ i.e. compulsory voting Pareto-dominates voluntary voting equilibrium $c_{m}$.

Proof Proposition 5. From (3.7), we have

$$
B_{M}(p)=(1-p)(q-0.5), B_{D}(p)=(1-2 \lambda)(q-0.5)
$$

and so from the definition of $\Phi_{S}(p)$ that

$$
\begin{aligned}
\Phi_{S}(p) & =0.5 F\left(B_{M}(p)\right)+0.5 F\left(B_{D}(p)\right) \\
& =(1-p)(q-0.5)(1-\lambda)
\end{aligned}
$$

So, assuming an interior solution $0<c_{M}^{*}, c_{D}^{*}<1, p^{*}$ is determined by $p^{*}=\Phi_{S}\left(p^{*}\right)$. Given (A.3), this can be solved to get

$$
p^{*}=\frac{(1-\lambda)(q-0.5)}{1+(1-\lambda)(q-0.5)}=p_{S}
$$

In the case where $\lambda>\hat{\lambda}$ so voting is according to private values, the benefit to voting, applying formula (3.2), is

$$
B_{P}(p)=\lambda\left(p B_{P}(1)+(1-p) B_{P}(0)\right)=\lambda(p 0.25+(1-p) 0.5)
$$

Equating this to $p$ and solving gives

$$
p^{*}=\frac{0.5 \lambda}{1+0.25 \lambda}=p_{P V}
$$

Finally, evaluating at $p_{S}, p_{P V}$ at $\lambda=\hat{\lambda}$;

$$
p_{P V}=\frac{0.5(q-0.5)}{q+0.25(q-0.5)}>p_{S}=\frac{0.5(q-0.5)}{q+0.5(q-0.5)}
$$

This completes the proof. 
Figure 1: Multiple Symmetric Bayesian Equilibria

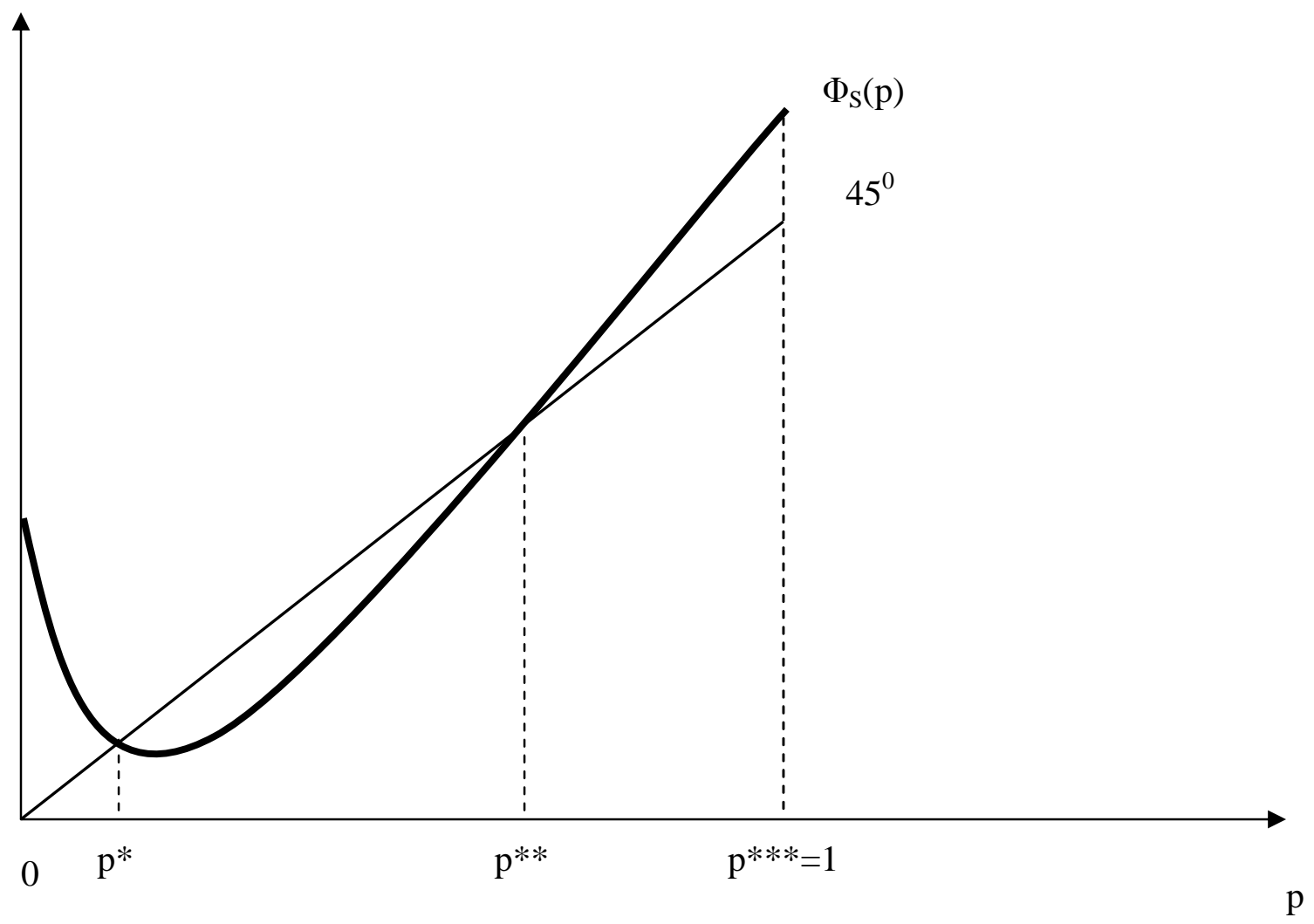


Figure 2: The Equilibrium Turnout Probability as Preference Bias Varies

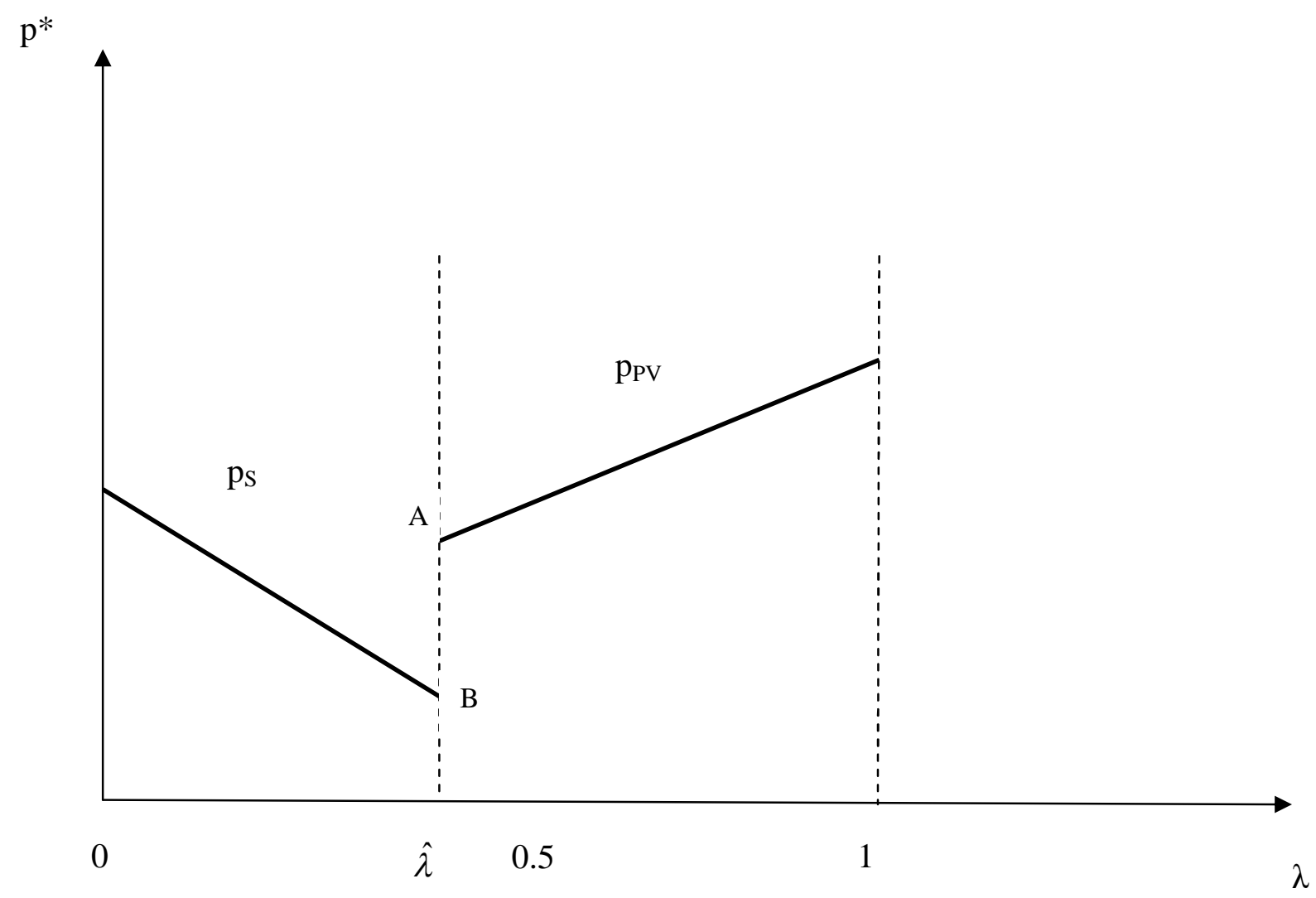


Table 1: Effect of Changes in q on Equilibrium Turnout Probability and Welfare*

\begin{tabular}{|l|r|r|r|r|r|r|r|r|r|}
\hline $\mathrm{q}$ & 0.71 & 0.72 & 0.73 & 0.74 & 0.75 & 0.76 & 0.77 & 0.78 & 0.79 \\
\hline $\mathrm{p}_{\mathrm{s}}$ & & & & & 0.143 & 0.148 & 0.153 & 0.157 & 0.162 \\
\hline $\mathrm{p}_{\mathrm{pv}}$ & 0.154 & 0.154 & 0.154 & 0.154 & 0.154 & & & & \\
\hline $\mathrm{EW}_{\mathrm{s}}$ & & & & & 0.537 & 0.539 & 0.542 & 0.545 & 0.548 \\
\hline $\mathrm{EW}_{\mathrm{pv}}$ & 0.512 & 0.512 & 0.512 & 0.512 & 0.512 & & & & \\
\hline
\end{tabular}

*A blank cell indicates that $\mathrm{p}$ or EW is not defined for that value of $\mathrm{q}$ 